\title{
Lepton and photon reconstruction and identification performance in ATLAS and CMS
}

\author{
Luigi Marchese* \\ on behalf of the ATLAS and CMS collaborations \\ University of Oxford \\ E-mail: luigi.marchese@physics.ox.ac.uk
}

This paper summarises lepton and photon performance results obtained by the ATLAS and CMS experiments during the Run-2 operations of the Large Hadron Collider (LHC) focusing on muons, electrons and photons. Such particles are key to important LHC physics results, including the Higgs boson discovery, precision measurements of Standard Model processes (SM) and searches for new physics beyond the SM. The paper first describes the reconstruction and identification efficiencies and the isolation studies for the two experiments. For electrons and photons the energy calibration strategy is presented, while for muons results on the muon momentum scale and resolution are reported. Even more than Run-1 data-taking operations, Run-2 operations are characterized by challenging pile-up conditions. Both the experiments are able to cope with higher rates.

7th Annual Conference on Large Hadron Collider Physics - LHCP2019

20-25 May, 2019

Puebla, Mexico

${ }^{*}$ Speaker. 


\section{The ATLAS and CMS experiments}

The ATLAS and CMS detectors at the CERN Large Hadron Collider (LHC) are general purpose devices designed primarily to probe proton-proton and heavy ion (proton-ion and ion-ion) collisions, including measurement and searches for new physics. They both consist of sub-detectors arranged in an onion-like cylindrical structure. Despite some differences, the two main layouts, when moving outwards from the interaction point, consist of:

- A tracker close to the interaction point which provides precise coordinate measurements of the tracks which are also used for triggering.

- Electromagnetic (ECAL) and hadronic calorimeters surrounding the tracker and providing measurements of the energy deposits of particles.

- A muon system which is the outermost part of both the detectors, reached only by muons and neutrinos. Together with the tracker it provides muon identification and track reconstruction, a trigger system and a measurement of the muon transverse momentum.

One of the most important differences between the ATLAS and CMS detectors comes from the magnetic field, which defines the size of the experiment. In ATLAS, a $2 \mathrm{~T}$ superconducting solenoid surrounds the Inner Detector (ID), the ATLAS tracker. In addition, a system of three large superconducting air-core toroid magnets, which gives a $0.5 \mathrm{~T}$ field strength in the barrel and $1 \mathrm{~T}$ in the endcaps provides the magnetic field for the Muon Spectrometer (MS) enabling improved measurements of the muon transverse momentum [1]. On the contrary, the magnetic field of the CMS detector is formed by a single $3.8 \mathrm{~T}$ superconducting solenoid surrounding the tracker and the calorimeters [2]. Differences in the magnet geometry result in a different size of the experiments. The CMS detector is a compact detector $21 \mathrm{~m}$ long with a diameter of $15 \mathrm{~m}$, whilst the ATLAS detector is a larger apparatus, extending $44 \mathrm{~m}$ in length with a diameter of $25 \mathrm{~m}$.

\section{Electron and photon performance}

Only information from the tracker and the ECAL is used to reconstruct and identify electrons and photons. Topologically connected clusters are defined to collect calorimeter signals of a given collision event in order to extract the significant signal from a background of electronic noise and other sources of fluctuations such as pile-up. For both electrons and photons, the application of a set of track- and cluster-quality criteria defines different categories targeting specific signal efficiencies and/or background rejections. They are better known as Working Points (WPs) and they will be used in the following sections.

\subsection{Electron reconstruction and identification efficiencies}

In ATLAS, an initial match between tracks from the Inner Detector with energy clusters in the ECAL is performed to select electron candidates. Calorimeter clusters benefit from a novel technique. At the beginning of Run 2, clusters were formed using a sliding window algorithm with fixed size. Recently, a dynamic, topological cell clustering-based approach has been adopted [3] [4]. The new strategy allows for improved measurements of electron and photon energies, with a 
special focus on cases where an electron radiates a bremsstrahlung photon, or a photon converts to an $e^{+} e^{-}$pair. The main reason preventing the application of the new technique in past was the inability to apply the simultaneous calibration strategy to the dynamical-sized clusters. The advent of new multivariate techniques has made this possible allowing for a new energy calibration, where regression decision trees are trained to correct the uncalibrated energy of electrons and photons.

When reconstructing electrons in ATLAS, in order to reject contamination by converted photons, pairs of tracks in the innermost layer of the silicon detector, the Insertable B-Layer, are not kept if they lead to a very small invariant mass. The electron identification relies on sequential and multivariate algorithms to reject electrons from photon conversions, hadronic jets or semileptonic hadron decays. A likelihood-based (LH) identification is implemented to select prompt electrons in the central region of the ATLAS detector $(|\eta|<2.47)$. Variables used in the LH include the longitudinal and lateral shower profiles, the electron's track conditions and the track-to-cluster matching. Combining all these different variables in a LH-based identification recovers electron candidates which fail a cut-based identification because they don't satisfy a selection criteria for a single quantity.

Similarly, in CMS electrons are reconstructed by associating a track in the silicon detector with a cluster of energy in the ECAL. Contiguous clusters in the ECAL are grouped into superclusters, composed of several clusters in the azimuthal-angle direction $\phi$. Several strategies are implemented to identify prompt isolated electrons, distinguishing them from background sources [5]. Complex and robust algorithms have been developed to combine several variables in an multivariate analysis to achieve a satisfying discrimination. The list of discriminating variables include observables for track-cluster matching, purely calorimeter observables and tracking observables to improve the separation with charged hadrons.

The tag-and-probe method based on the decay channels $Z \rightarrow e e$ and $J / \psi \rightarrow e e$ is used to measure the electron reconstruction efficiencies. The efficiencies of the LH-based electron identification for the Tight, Medium and Loose operating WPs as a function of the transverse Energy, $E_{T}$, for ATLAS are shown in Figure 1(left). Overall, trends are similar with efficiencies in the range $55 \%$ at $E_{T}=4.5 \mathrm{GeV}$ and $90 \%$ at $E_{T}=100 \mathrm{GeV}$ for the Tight WP, achieving even $96 \%$ at $E_{T}=$ $100 \mathrm{GeV}$ for the Loose WP. Similarly, efficiencies for the Loose WP as a function of transverse momentum of the electron candidate are presented in Fig. 1(right) for CMS. Only $Z \rightarrow e e$ candidates are used in this figure.

\subsection{Photon reconstruction and identification efficiencies}

Photon reconstruction closely follows electron reconstruction. The only difference is that no tracks are expected in the tracker, unless for photon conversion where a photon converts to an electron-positron pair, in which case the corresponding tracks need to be retrieved.

In ATLAS, information from the calorimeters is used to define the identification WPs. Prompt photons are separated from background photons by implementing a selection on quantities describing the shape and properties of the related electromagnetic shower and by imposing isolation criteria for the photon candidate [7], as described in Sec. 2.3. Fig. 2(left) shows the identification efficiency of the Tight identification requirement for unconverted photons from radiative $Z \rightarrow e^{+} e^{-} \gamma$ and $Z \rightarrow \mu^{+} \mu^{-} \gamma$ decays as a function of the number of interactions per bunch crossing, $\mu$, averaged over pseudorapidity. Results are shown for the Loose isolation operating WP. Data 

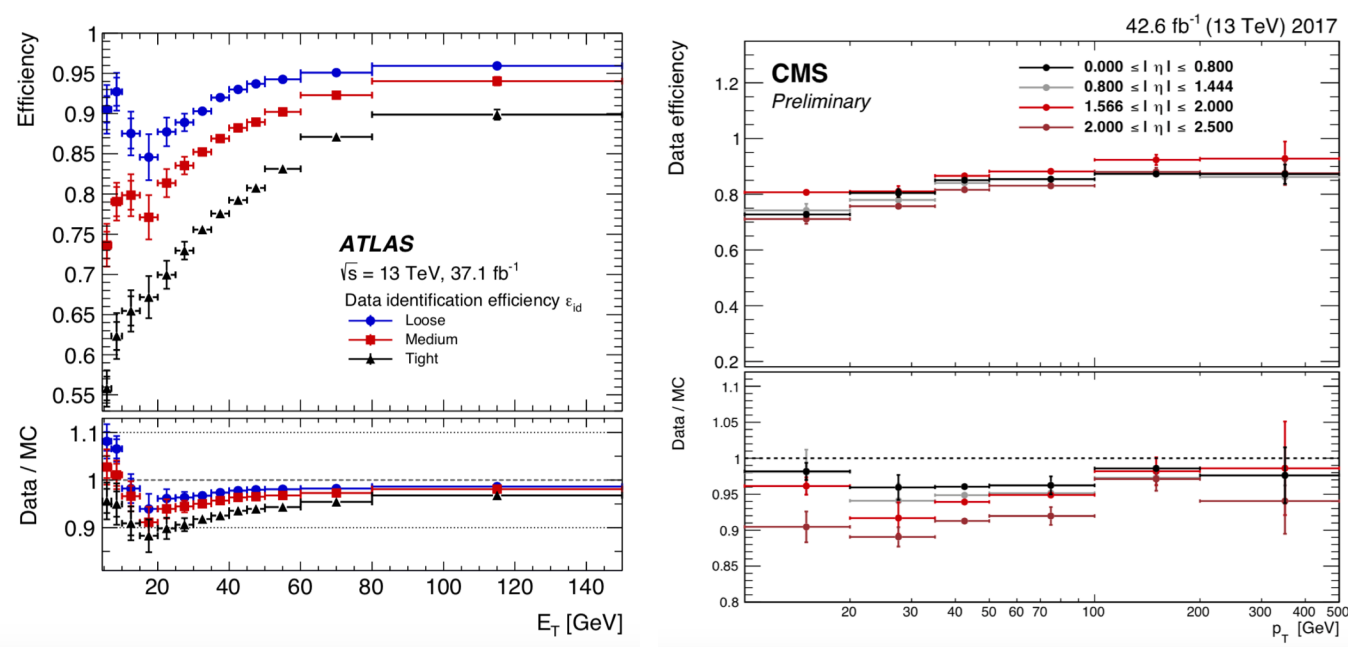

Figure 1: (Left) electron identification efficiency for data and the ratio to simulation (lower pad) as a function of the transverse energy, $E_{T}$, for the Tight (black markers), Medium (red markers) and Loose (blue markers) operating Working Points WPs in ATLAS [4]; (Right) electron identification efficiency for data and the ratio to simulation (lower pad) for the Loose operating WP as a function of the electron transverse momentum for different pseudorapidity ranges in CMS [6]. Isolation criteria are included, as discussed in Sec. 2.2.1.

are compared to the simulation showing a good agreement. High efficiency is observed also for high values of $\mu$.

In CMS, the identification WPs are very similar to the ones for electrons, with some differences in the cuts and selection criteria. In addition, photons traversing the CMS tracker are extremely likely to convert into electron-positron pairs. The simulation estimates that about $60 \%$ of photon conversions occur before the last three layers of the tracker, in regions with the largest amount of tracker material in front of the ECAL. Although converted photons are fully reconstructed in the ECAL, improvements are gained by reconstructing the associated $e^{+} e^{-}$track pair [6]. Fig. 2(right) shows the photon identification efficiency for the multivariate identification (Boosted Decision Trees) WP of $90 \%$ of signal efficiency as a function of the photon transverse momentum for different pseudorapidity ranges. The efficiency is measured with the tag-and-probe method using $Z \rightarrow e e$ decays and electrons reconstructed as photons.

\subsubsection{Electron isolation efficiencies}

In order to differentiate the prompt production of electrons, muons and photons in signal processes, for instance decays of heavy resonances like Higgs, $W$ and $Z$ bosons, from background processes, isolation criteria are implemented. Signal events are characterized by a specific signature, i.e. low activity (both in the calorimeter and in the tracker) in an area $\Delta \eta \times \Delta \phi$ surrounding the trajectory of the candidate object. The activity in the vicinity of the candidate object, for instance an electron, is studied by summing the transverse energy of clusters in the calorimeter (calorimeter-based isolation) or the transverse momenta of tracks (track-based isolation) in a cone of radius $\Delta R=\sqrt{(\Delta \eta)^{2}+(\Delta \phi)^{2}}$ around the electron direction, having subtracted the contribution of the electron itself. Both calorimeter- and track-based isolation variables are used in ATLAS. WPs are then defined consisting of cuts applied on the two isolation variables. Depending on the 

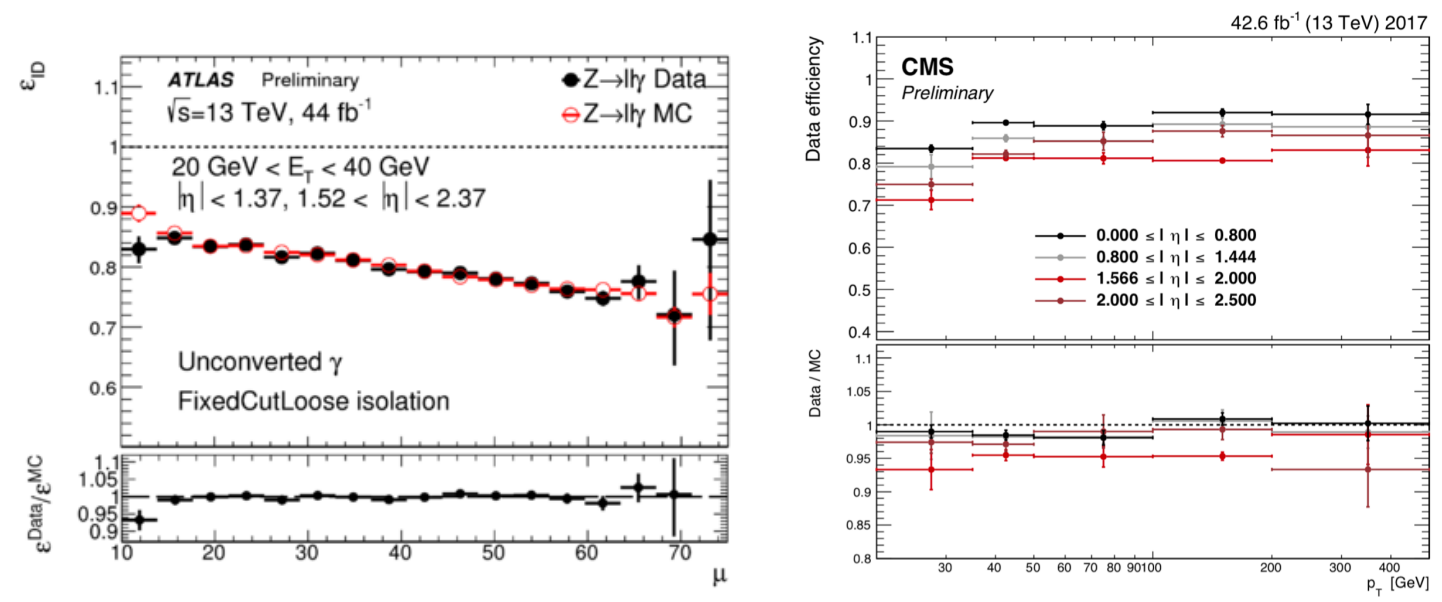

Figure 2: (Left) Photon efficiency of the Tight identification requirement for unconverted photons from radiative $Z$ decays as a function of the number of interactions per bunch crossing, $\mu$, averaged over pseudorapidity in ATLAS [8]. Loose photon isolation requirements are applied, as described in Sec. 2.3. Only the statistical uncertainties are shown. Data/MC is also shown (lower pad); (Right) Photon identification efficiency for data and the ratio to simulation (lower pad) for the multivariate identification (Boosted Decision Trees) working point of $90 \%$ of signal efficiency as a function of the photon transverse momentum for different pseudorapidity ranges in CMS [6]. Isolation criteria are included, as discussed in Sec. 2.3. The efficiency is measured with the tag-and-probe method using $Z \rightarrow e e$ decays and electrons reconstructed as photons.

signal efficiency or purity one wants to achieve, the cut values can be fixed or vary as a function of $E_{T}$. In Figure 3 the isolation efficiency for the various Fixed WPs is shown as a function of $E_{T}$ in $Z \rightarrow e e$ events.

In CMS, two isolation techniques are available. The simplest one is detector-based. Similar to the ATLAS approach, it relies on the definition of a cone around each electron trajectory in the calorimeters and in the tracker. In addition, since a particle flow (PF) algorithm [9] combines information from more sub-detectors, PF-based isolation quantities are defined. In this second case, isolation is defined using the PF candidates reconstructed with a momentum direction within an isolation cone. The main advantage is the possibility to use the correct calibration for each object, avoiding a possible double-counting of energy deposits assigned to particle candidates. In contrast to the ATLAS requirements, the CMS isolation criteria are included in the identification WPs for both electrons and photons.

\subsection{Photon isolation efficiencies}

Prompt photons are defined as not originating from hadron decays and are produced via several mechanisms in the Standard Model (SM), such as parton-parton scattering ( $q g, q \bar{q} \rightarrow \gamma+X, g g$, $q \bar{q} \rightarrow \gamma \gamma$ ), QED radiation, $H \rightarrow \gamma \gamma$. Background photons can originate from hadronic jets, produced with significant cross sections or from hadrons mis-identified as photons. Because of the way they are produced, background photons are generally surrounded by sizable hadronic activity in their vicinity. Therefore, good isolation criteria can significantly suppress most of them. As done for electrons and muons, isolation criteria are defined based on the transverse energy flow in a cone 


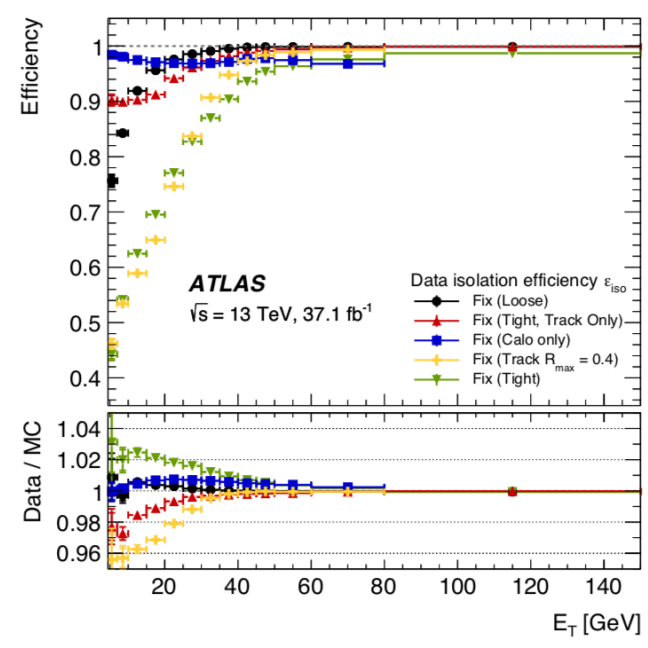

Figure 3: Isolation efficiency for data and the ratio to simulation (lower pad) for the various fixed operating Working Points WPs in ATLAS [8].

around the direction of the photon candidate. Track- and calorimeter-based isolation variables are defined. In CMS, as for electrons, PF-based isolation quantities are also defined.

Fig. 4 reports the identification efficiency of the Tight identification requirement for unconverted photons from radiative $Z \rightarrow e^{+} e^{-} \gamma$ and $Z \rightarrow \mu^{+} \mu^{-} \gamma$ decays as a function of transverse energy of the candidate, averaged over the pseudorapidity of the photon candidate in ATLAS. Results are shown for the Loose isolation operating WP. Data are compared to the simulation showing a good agreement.

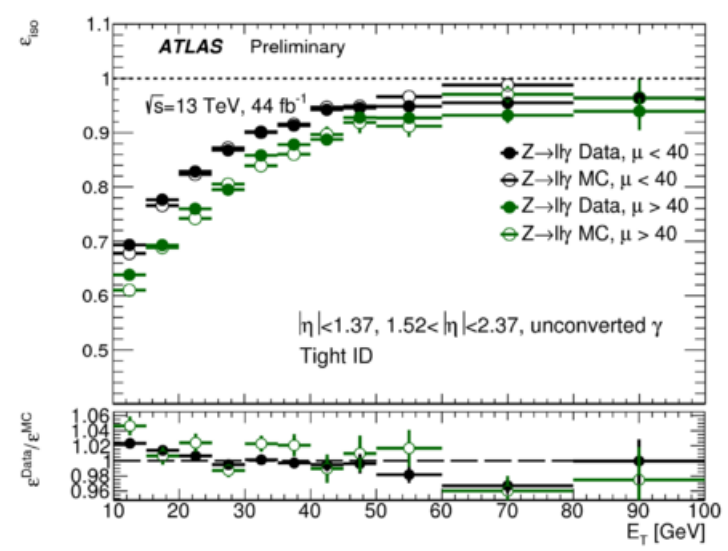

Figure 4: Isolation efficiency for data and the ratio to simulation (lower pad) for unconverted photons using fixed cut for the Loose operating WP in ATLAS. Results are derived from radiative $Z \rightarrow e^{+} e^{-} \gamma$ and $Z \rightarrow \mu^{+} \mu^{-} \gamma$ decays as a function of transverse energy of the candidate, averaged over the pseudorapidity of the photon candidate [8].

\subsection{Electron and photon energy calibration}

A precision calibration of the electron and photon energy is needed for precision measure- 
ments such as Higgs, $W$ or $Z$ mass and cross-section measurements. In ATLAS the calibration procedure consists of several steps. A simulation-based calibration is applied to data and to the simulation using a multivariate-based algorithm with gradient boosting. Then, uniformity corrections in detector response are applied by implementing some data-driven corrections based solely on data. Subsequently, the overall energy scale in data is adjusted and, based on the data/MC difference in energy resolution, a MC correction is also evaluated and applied to the simulation. Such a correction is universal, working for both electrons and muons. Results are finally validated by comparing data and MC [10].

A similar calibration procedure is also implemented in CMS with the energy corrected to account for energy containment effects. Corrections are separately tuned for electrons and photons [6].

Results of the calibration are shown in Fig. 5 for both the collaborations. A good data/MC agreement is achieved in both cases. The two experiments show similar results in terms of resolution which is at the percent level in the barrel.
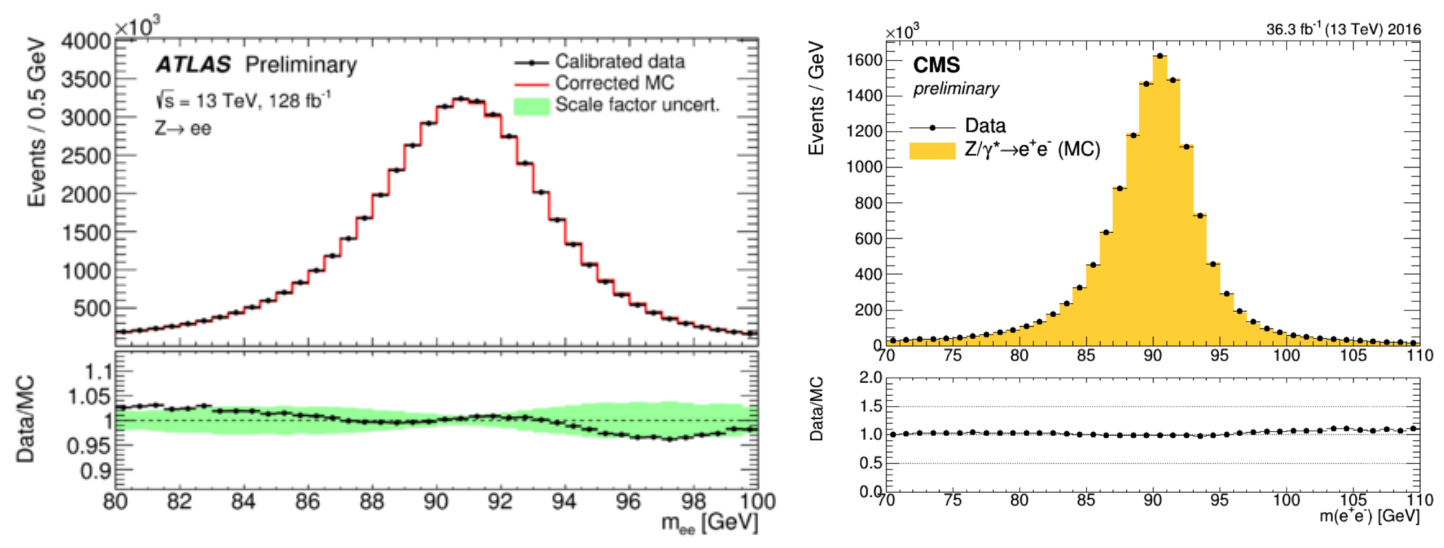

Figure 5: Results of the electron energy calibration in ATLAS (Left) [11] and CMS (Right) [6] when looking at the invariant-mass distribution in $Z \rightarrow e e$ events after the applications of the derived corrections.

\section{Muon performance}

Information from all the ATLAS and CMS sub-detectors is used to reconstruct and identify muons.

\subsection{Muon reconstruction and identification efficiencies}

In ATLAS muon reconstruction is performed independently in the Inner Detector and Muon Spectrometer. The individual-detector information is then combined to form combined muon tracks corresponding to about $96 \%$ of all muons used in physics analyses. Other muon types are possible, such as calorimeter-tagged muons, based on calorimeter-energy deposit match with ID tracks. These less pure muon types are mostly used to recover acceptance in the regions where the ATLAS MS is only partially instrumented to allow for cabling and services to calorimeters and the ID. Several WPs are defined based on criteria on the muon type, including track-quality and the 
charge-over-momentum matching between the ID and MS track. These WPs are required to address the various needs of different physics analyses, targeting specific efficiencies and purity rates for the identification selection [12].

Similar to ATLAS, muon reconstruction in CMS can start from the Tracker propagating to the Muon System or vice versa with an inside-out or outside-in algorithm defining Tracker and Global muon tracks, respectively. Tracker muon tracks have higher efficiency in regions of the CMS detector with less instrumentation and for low-momenta muons, while Global muon tracks are designed to have high efficiency for muons penetrating through more than one muon station, reducing the muon misidentification rate compared to Tracker muon tracks. Reconstructed muons are fed into the CMS PF algorithm which adds information from all the other CMS sub-detectors. PF applies a set of track-quality selections to reconstructed candidates with the standalone global or tracker muon algorithm. As a result, also in CMS various WPs are defined [13].

The tag-and-probe method is used, as done for electrons, to estimate the muon reconstruction efficiency. Muons from $Z \rightarrow \mu \mu$ and $J / \psi \rightarrow \mu \mu$ decays are used to cover the high- and lowmomentum spectrum, respectively. Results in data and MC are shown in Figure 6 for both the collaborations: (left) is the muon reconstruction efficiency for the various WPs in ATLAS, Tight, Medium and Loose, as a function of the muon pseudorapidity and based on $Z \rightarrow \mu \mu$ decays for muons with $p_{T}>10 \mathrm{GeV}$; (right) is the muon reconstruction efficiency as a function of the muon transverse momentum for the Tight WP in CMS. Efficiencies are above 95\% for muons with $p_{T}>$ $20 \mathrm{GeV}$.

Good data/MC agreement is observed in both the collaborations.
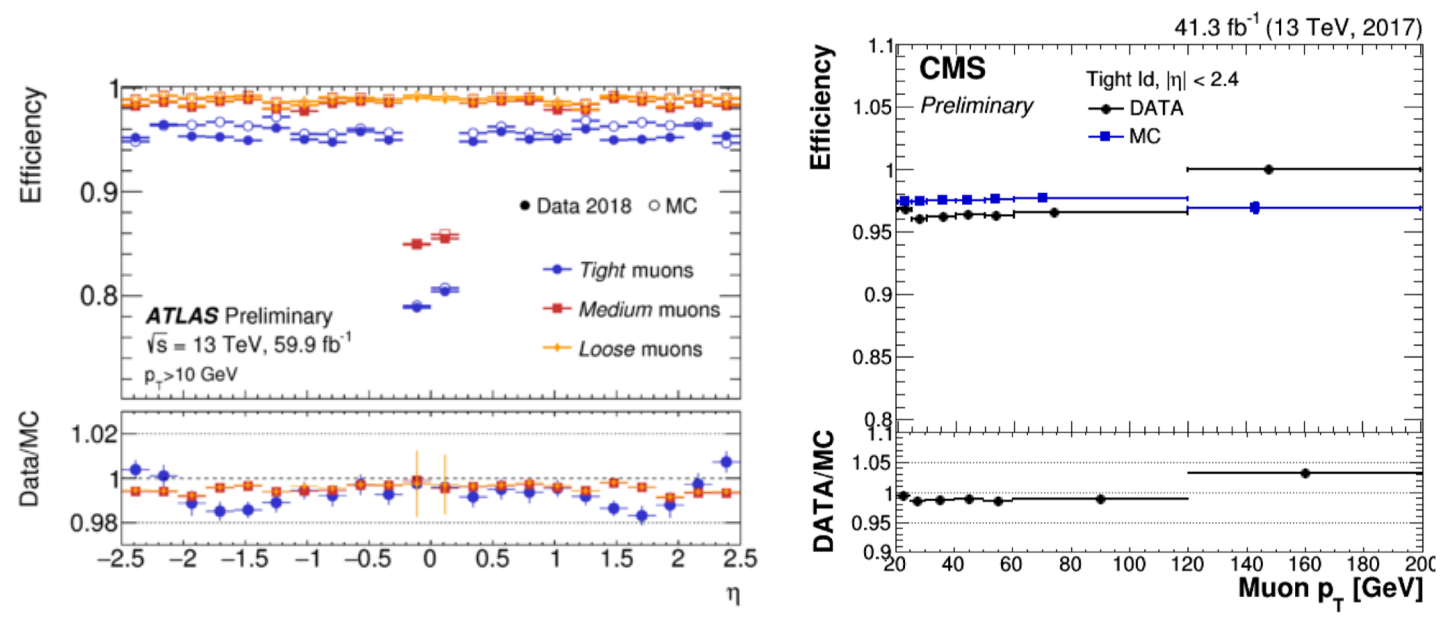

Figure 6: (Left) Muon reconstruction efficiency and the data/MC ratio (lower pad) as a function of the muon pseudorapidity for the Tight (blue markers), Medium (red markers) and Loose (green markers) operating WPs in ATLAS [14]; (Right) muon reconstruction efficiency and the data/MC ratio (lower pad) for the Loose operating WP as a function of the muon transverse momentum for the Tight WP in CMS [15].

\subsection{Muon isolation efficiencies}

For both collaborations, isolation studies are performed as done for electrons and photons. In Fig. 7 the isolation efficiency is shown for the fixed cut Loose WP as a function of the muon 
transverse momentum in ATLAS (left) and for the Tight PF isolation WP as a function of the muon pseudorapidity in CMS (right). Good data/MC agreement is observed. Results are derived using a sample of muons from $Z \rightarrow \mu \mu$ decays.

During Run-2 operations both LHC collaborations had to deal with high-pile-up events. In order to guarantee excellent isolation efficiencies for high values of pile-up events, an ad hoc optimization of the isolation selection has been performed in ATLAS. Fig. 8 show the results obtained using a tag-and-probe data-analysis of $Z \rightarrow \mu \mu$ decays. High isolation efficiency is maintained even at high $\mu$.
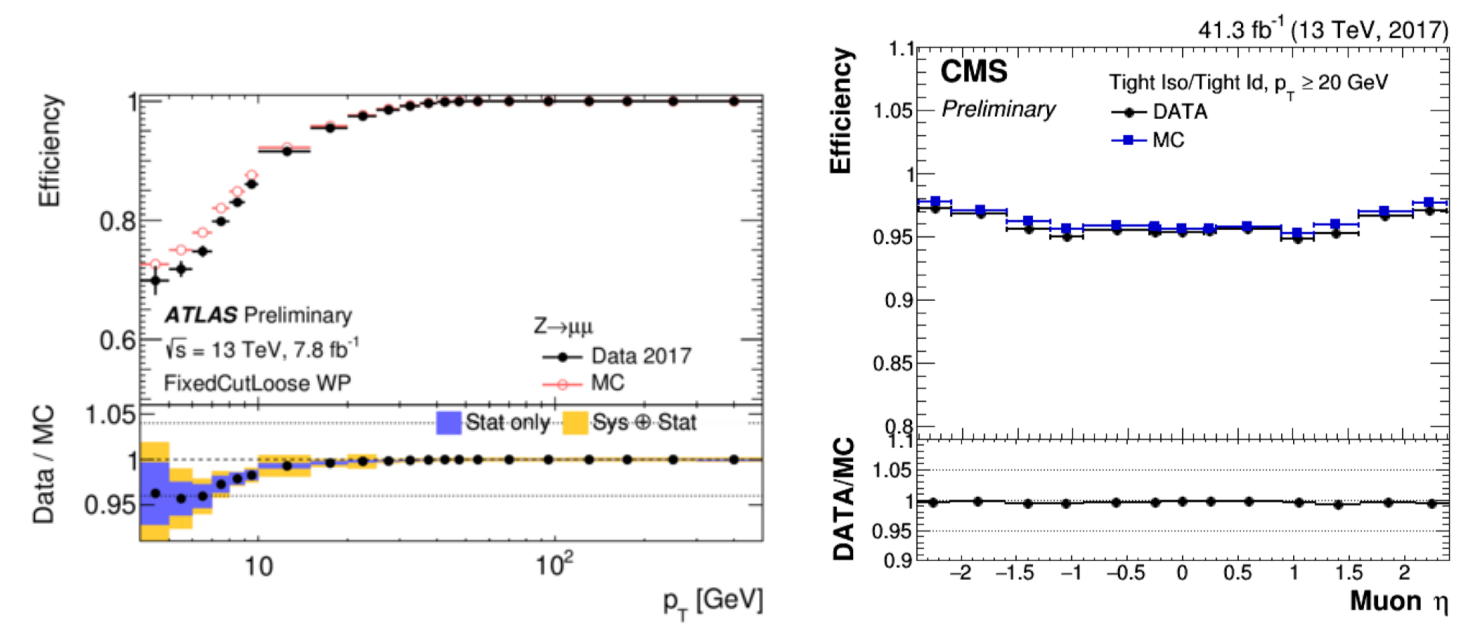

Figure 7: Muon isolation efficiency measured in $Z \rightarrow \mu \mu$ events for the fixed cut Loose WP as a function of the muon pseudorapidity in ATLAS (Left) [16] and for the Tight PF isolation WP as a function of the muon transverse momentum (Right) in CMS [15].

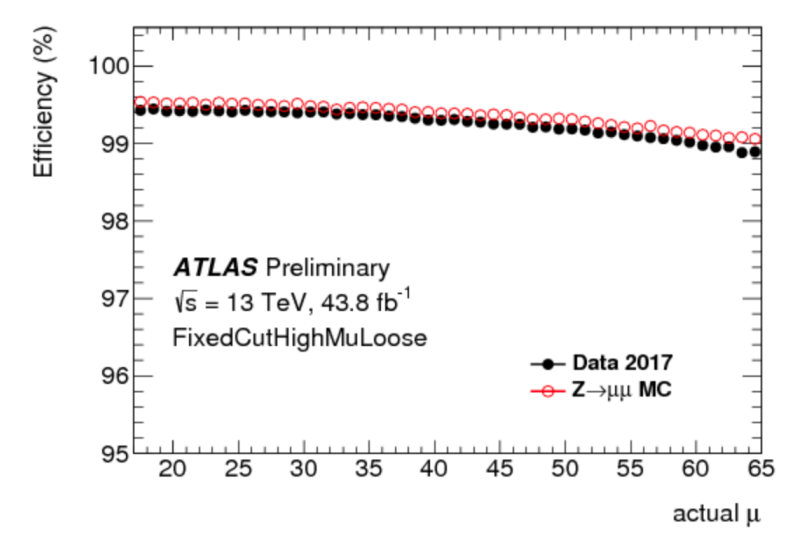

Figure 8: Selection efficiencies for different muon isolation requirements measured as a function of the pile-up in ATLAS [17].

\subsection{Muon Momentum scale and resolution}

Although the ATLAS simulation contains an accurate description of the detector in terms of material and geometry, this is not enough to reach the precision level needed by precision results. 
For measurements such as the Higgs boson mass, the muon momentum scale should be known at least at the per mill level and the muon momentum resolution at the percent level. To obtain such a precision, some corrections are applied to the MC muon momentum [12].

In ATLAS, corrections are extracted with an iterative procedure performing a binned maximumlikelihood fit with templates derived from simulation which compares the $Z \rightarrow \mu \mu$ and $J / \psi \rightarrow \mu \mu$ invariant mass spectra in data and MC. Corrections are later validated on muons from $\Upsilon \rightarrow \mu \mu$ [18] and from $Z \rightarrow \mu \mu$ and $J / \psi \rightarrow \mu \mu$ decays to cover the entire momentum range. Fig. 9 shows the data-to-MC comparison in $\Upsilon \rightarrow \mu \mu$ decays for ATLAS. Agreement at the per mil level for the scale and at the percent level for the resolution is reached in ATLAS.

In CMS, the momentum bias is fitted by linear and constant terms using $Z \rightarrow \mu \mu$ and $J / \psi \rightarrow$ $\mu \mu$ mass spectra for low and intermediate $p_{T}$ and cosmic rays at high $p_{T}$.
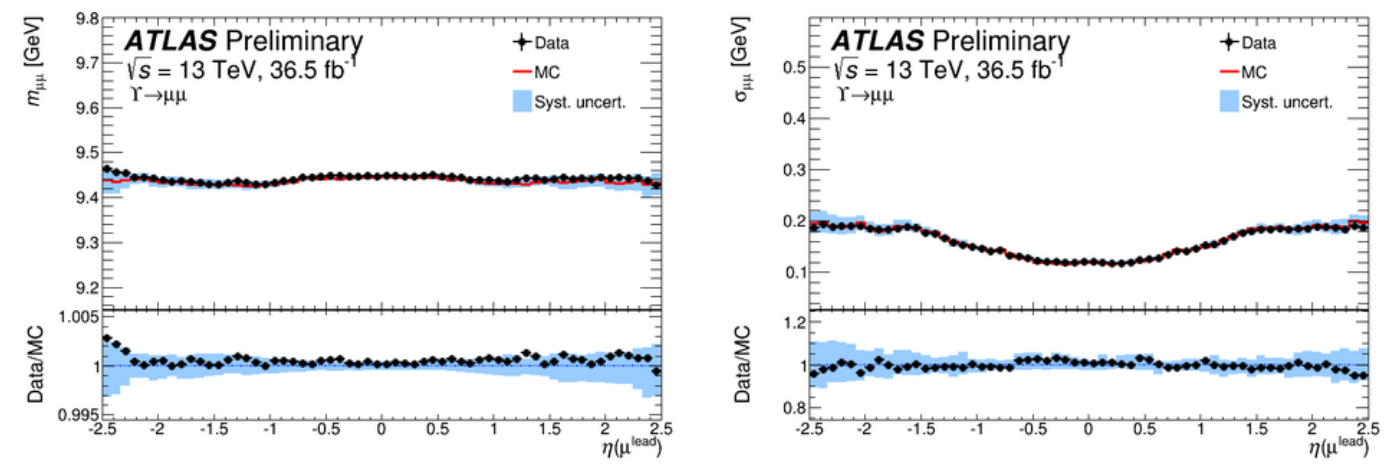

Figure 9: Muon momentum scale and resolution for combined muons, measured in $\Upsilon \rightarrow \mu \mu$ decays [18]. Results are shown as a function of the pseudorapidity, $\eta$, of the leading muon.

\section{Conclusions}

The performance of lepton and photon identification algorithms for the ATLAS and CMS experiments were reported using LHC Run-2 data. Results for reconstruction, identification and isolation efficiencies were presented in both data and MC, showing satisfying agreement. For muons results on the muon momentum scale and resolution were also reported. Despite the increasing number of pile-up events, excellent performance is still observed. The two collaborations differ for the used strategies due to differences in the detector technologies, but overall good and consistent results are observed.

\section{References}

[1] ATLAS Collaboration, J. Inst. 08 S08003, 3 (2008)

[2] CMS Collaboration, J. Inst. 08 S08004, 3 (2008)

[3] ATLAS Collaboration, ATL-PHYS-PUB-2017-022 (2017)

[4] ATLAS Collaboration, Eur. Phys. J. C 79 (2019) 639

[5] CMS Collaboration, CMS-DP-2017-004 (2017) 
[6] CMS Collaboration, CMS-DP-2018-017 (2018)

[7] ATLAS Collaboration, ATL-PHYS-PUB-2016-014 (2016)

[8] ATLAS Collaboration, EGAM-2018-007 (2018)

[9] CMS Collaboration, JINST 12 (2017) P10003

[10] ATLAS Collaboration, JINST 14 (2019) P03017

[11] ATLAS Collaboration, EGAM-2018-008 (2018)

[12] ATLAS Collaboration, Eur. Phys. J. C 76 (2016) 292

[13] CMS Collaboration, JINST 13 (2018) P06015

[14] ATLAS Collaboration, MUON-2018-007 (2018)

[15] CMS Collaboration, CMS-DP-2018-042 (2018)

[16] ATLAS Collaboration, MUON-2017-002 (2017)

[17] ATLAS Collaboration, MUON-2018-002 (2018)

[18] ATLAS Collaboration, ATL-PHYS-PUB-2019-018 (2019) 